\title{
Applications of Liquid Anion Exchangers for the Separation of Zirconium and Hafnium
}

\author{
Manjusha A. KARVE and Shripad M. KHOPKaR \\ Department of Chemistry, Indian Institute of Technology, Bombay 400 076, India
}

\begin{abstract}
Zirconium and hafnuim were extracted with $0.1 \mathrm{M}$ of Aliquat $336 \mathrm{~S}$ in toluene from $0.005 \mathrm{M}$ of ascorbic acid at pH 4.0. Both of the metals were stripped with $2 \mathrm{M}$ hydrochloric acid. Zirconium from the aqueous phase was determined spectrophotometrically with Arsenazo III at $650 \mathrm{~nm}$, while hafnium was determined with Xylenol Orange at $540 \mathrm{~nm}$. The separation of zirconium from hafnium was achieved by stripping hafnium with $9 \mathrm{M}$ hydrochloric acid followed by stripping zirconium with $2 \mathrm{M}$ hydrochloric acid. They were also separated from associated elements in multicomponent mixtures.
\end{abstract}

Keywords Zirconium, hafnium, solvent extraction, Aliquat 336S, ascorbic acid

Because of lanthanide contraction, the chemical behavior of zirconium and hafnium is similar and, hence, the separation of zirconium from hafnium is somewhat difficult. Solvent-extraction methods, however, are most effective for the purpose of separation.

The separation of zirconium and hafnium was carried out with $60 \%$ tributyl phosphate ${ }^{1}$ from either $6 \mathrm{M}$ nitric acid or a thiocyanate media with isobutyl methyl ketone ${ }^{2}$ or polyurethane ${ }^{3}$ as extractants. $\beta$-Diketones ${ }^{4}$ were also used. High molecular-weight amines were used in chloride $^{5}$ or sulfate media with Primene JMT or Aliquat $336 \mathrm{~S}^{6}$ used as the extractant.

Unfortunately, systematic investigations from organic acid solutions have been limited. Such a separation was effected from malonic acid with $0.05 \mathrm{M}$ Amberlite LA-2 in xylene. ${ }^{7}$ The citrate buffered at $\mathrm{pH} 3.0-6.0$ was also used with Amberlite LA-1. ${ }^{8}$ Separation from salicylate media was unsatisfactory, since it invloved both masking and demasking reactions. ${ }^{9}$ Since studies invloving ascorbic acid have been lacking, and endeavor was made to devise the separation of zirconium from hafnium from ascorbic acid with Aliquat $336 \mathrm{~S}$ used as the extractant. It was possible to separate zirconium from hafnium as well as several other elements.

\section{Experimental}

\section{Apparatus and reagents}

The apparatus used was similar to that described earlier. ${ }^{10}$ A stock solution of zirconium was prepared by dissolving $2.50 \mathrm{~g}$ of zirconium nitrate pentahydrate (BDH, AnalR) in $50 \mathrm{ml}$ of concentrated nitric acid. This solution was diluted to $500 \mathrm{ml}$ with distilled water, and standardized complexometrically. ${ }^{11}$ It contained
$1 \mathrm{mg} / \mathrm{ml}$ of zirconium. A diluted solution containing $40 \mu \mathrm{g} / \mathrm{ml}$ of zirconium was prepared by twenty-five fold dilution.

A stock solution of hafnium was prepared by dissolving $1.5 \mathrm{~g}$ of hafnium dioxide (BDH) in a mixture of hydrofluoric acid and sulfuric acid $(1: 1 \mathrm{v} / \mathrm{v})$ in a platinum crucible. The dissolved solution was diluted with sulfuric acid and made up to $500 \mathrm{ml}$ with distilled water. It was standardized gravimetrically with $N$-benzoyl- $N$ phenylhydroxyl amine. ${ }^{11}$ It contained $2.54 \mathrm{mg} / \mathrm{ml}$ hafnium. A diluted solution containing $25.4 \mu \mathrm{g}$ of hafnium was prepared by hundred-fold dilution. Aliquat 336S (General Mills Ltd., UK), Amerlite LA-1 or Amerlite LA-2 or Primene JMT (Rohm and Hass Co., USA), trioctylamine (Riedel Haen, Germany) was used in the ascorbate form.

\section{General procedure}

To an aliquot of a solution containing $40 \mu \mathrm{g}$ of either zirconium or hafnium, $1 \mathrm{ml}$ of a $0.005 \mathrm{M}$ ascorbic acid solution was added. The $\mathrm{pH}$ of the resulting solution was adjusted to 4.0 with either $0.001 \mathrm{M}$ ammonium hydroxide or $0.001 \mathrm{M}$ ascorbic acid. In actual practice, less than $1 \mathrm{ml}$ of these solutions were required for the $\mathrm{pH}$ adjustment. The solution was made up to $10 \mathrm{ml}$ with distilled water; it was then transferred to a separatory funnel and shaken with $10 \mathrm{ml}$ of $0.1 \mathrm{M}$ Aliquat $336 \mathrm{~S}$ in toluene for $10 \mathrm{~min}$ on a wrist-action flask shaker. The two phases were allowed to settle and separate. From the organic phase, either zirconium or hafnium was stripped with $2 \mathrm{M}$ hydrochloric acid. The zirconium was then determined spectrophotometrically in the aqueous phase as its complex with Arsenazo III ${ }^{11}$ at $660 \mathrm{~nm}$; the hafnium was determined from the aqueous phase with Xylenol Orange $^{11}$ at $540 \mathrm{~nm}$. 


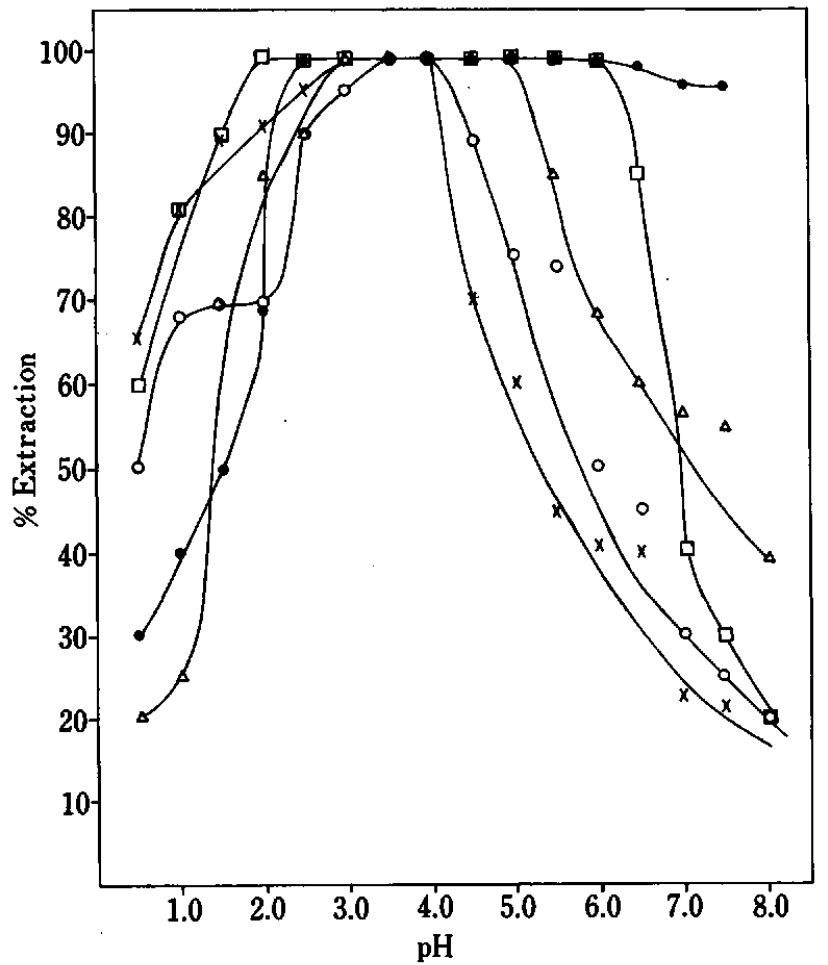

Fig. 1 Extraction of zirconium as a function of $\mathrm{pH}$ with various liquid anion exchangers. $0,0.1 \mathrm{M}$ Aliquat $336 \mathrm{~S} ; \triangle$, 0.1 M Amberlite LA-1; O, $0.08 \mathrm{M}$ Amberlite LA-2; $\times, 0.1 \mathrm{M}$ trioctylamine; $\square, 0.1 \mathrm{M}$ Primene JMT.

\section{Results and Discussion}

The optimum $\mathrm{pH}$ for a quantitative extraction of zirconium or hafnium was ascertained by extracting between $\mathrm{pH} 0.5-8.0$ with various liquid anion exchangers (Figs. 1 and 2). Zirconium was quantitatively extracted with $0.1 \mathrm{M}$ each Aliquat $336 \mathrm{~S}$ at (pH 2.5-6.5), Amberlite LA-1 (pH 3.0-5.0), Amberlite LA-2 $(\mathrm{pH} 3.5-4.0)$, trioctylamine $(\mathrm{pH} 3.0-4.0)$ and Primene JMT (pH 2.0-6.0). However, the extraction of hafnium was quantitative only with $0.1 \mathrm{M}$ Aliquat $336 \mathrm{~S}$ (pH 3.0 - 7.5). Thus, $0.1 \mathrm{M}$ Aliquat 336S was preferred as the extractant for zirconium as well as for hafnium at pH 4.0.

Extraction as a function of the Aliquat 336 S concentration Zirconium or hafnium was extracted at $\mathrm{pH} 4.0$ with 0.01 to $0.1 \mathrm{M}$ of Aliquat $336 \mathrm{~S}$ in toluene (Table 1). Zirconium was extracted with 0.07-0.1 M Aliquat 336S, while hafnium was extracted with $0.06-0.1 \mathrm{M}$ of Aliquat 336S. Hence, $0.1 \mathrm{M}$ Aliquat $336 \mathrm{~S}$ in toluene was used for both metals.

\section{Extraction as a function of the ascorbic acid concentration}

With other factors kept constant, zirconium or hafnium was extracted from $0.0005-0.005 \mathrm{M}$ of ascorbic acid as the complexing agent. The optimum concent-

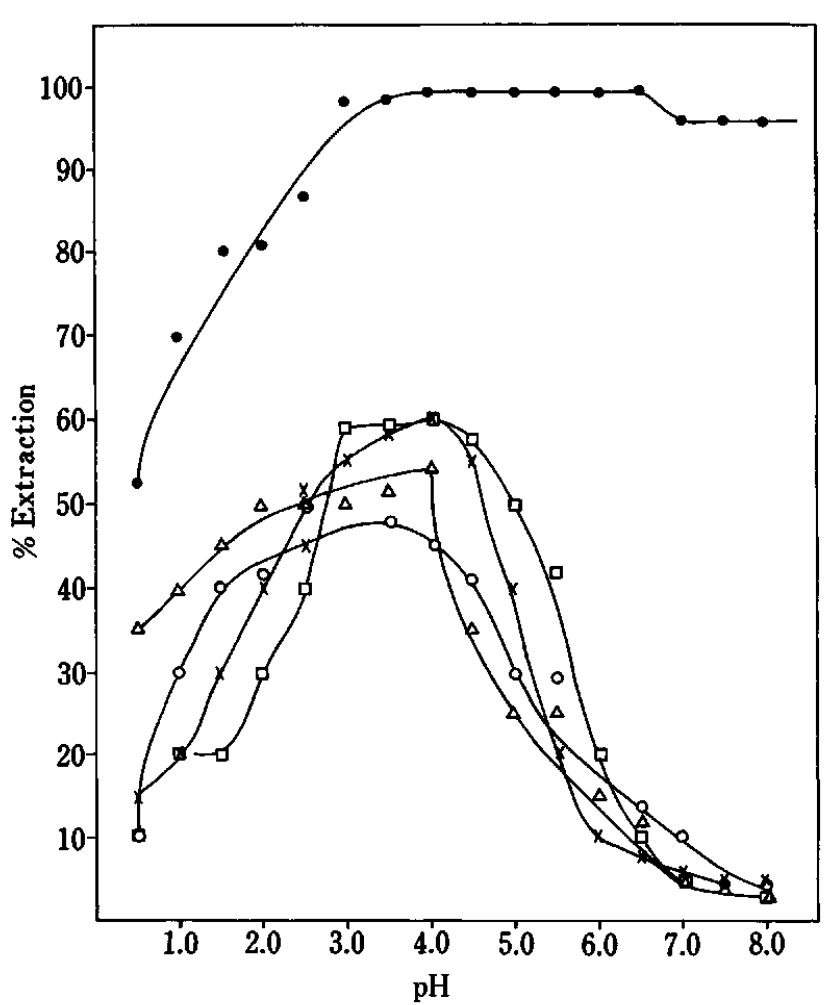

Fig. 2 Extraction of hafnium as a function of $\mathrm{pH}$ with various liquid anion exchangers. $\quad, 0.1 \mathrm{M}$ Aliquat $336 \mathrm{~S} ; \triangle, 0.1 \mathrm{M}$ Amberlite LA-1; O, $0.08 \mathrm{M}$ Amberlite LA-2; $\times, 0.1 \mathrm{M}$ trioctylamine; $\square, 0.1 \mathrm{M}$ Primene JMT.

Table 1 Effect of the Aliquat $336 \mathrm{~S}$ concentration

\begin{tabular}{lccccc}
\hline \multirow{2}{*}{ Aliquat 336S/M } & \multicolumn{2}{c}{ Zirconium } & & \multicolumn{2}{c}{ Hafnium } \\
\cline { 2 - 3 } \cline { 5 - 6 } & $\% E$ & $D$ & & $\% E$ & $D$ \\
\hline 0.01 & 54.0 & 1.1 & & 60.0 & 1.5 \\
0.02 & 56.0 & 1.2 & & 64.0 & 1.7 \\
0.03 & 65.1 & 1.8 & & 70.0 & 2.3 \\
0.04 & 79.0 & 3.7 & & 79.0 & 3.7 \\
0.05 & 85.2 & 5.7 & & 85.0 & 5.6 \\
0.06 & 92.1 & 11.6 & & 99.0 & 99 \\
0.07 & 99.7 & 332.6 & & 99.0 & 249 \\
$0.08-0.1$ & 99.7 & $>332$ & & 99.6 & $>249$ \\
\hline
\end{tabular}

Table 2 Effect of the ascorbic acid concentration

\begin{tabular}{llllll}
\hline \multirow{2}{*}{ Ascorbic acid/M } & \multicolumn{2}{c}{ Zirconium } & & \multicolumn{2}{c}{ Hafnium } \\
\cline { 2 - 3 } \cline { 5 - 6 } & $\% E$ & $D$ & & $\%$ & $D$ \\
\hline 0.0005 & 42.0 & 0.72 & & 30.0 & 0.42 \\
0.0006 & 50.0 & 1.0 & & 40.0 & 0.66 \\
0.0007 & 59.1 & 1.4 & & 51.0 & 1.0 \\
0.0008 & 62.0 & 1.6 & 59.0 & 1.4 \\
0.0009 & 66.8 & 2.0 & 62.0 & 1.6 \\
0.001 & 75.0 & 3.3 & 70.0 & 2.3 \\
0.002 & 82.1 & 4.5 & 75.0 & 3.0 \\
0.003 & 91.0 & 10.1 & 86.0 & 6.1 \\
0.004 & 95.6 & 21.7 & & 95.0 & 19.0 \\
$0.005-0.05$ & 99.7 & $>332$ & 99.6 & $>249$ \\
\hline
\end{tabular}


ration of ascorbic acid for the extraction of zirconium, as well as for hafnium, was $0.005 \mathrm{M}$ (Table 2).

\section{Effect of diluents}

Various nonpolar solvents (benzene, toluene, xylene, chloroform and carbon tetrachloride) were used as the diluents (Table 3). The volume of the organic-toaqueous phase was maintained at $1: 1$ in order to avoid any third-phase formation and emulsification. Zirconium was quantitatively extracted with benzene, toluene and xylene as the diluents, while hafnium was extracted with toluene and xylene. Due to the non-toxicity nature and absence of emulsification, and a clear-cut phase separation, toluene was preferred as the diluent for both metals.

\section{Extraction as a function of the stripping agents}

Zirconium or hafnium was stripped after extraction with $0.01-10 \mathrm{M}$ of various mineral acids (Table 4). Zirconium was stripped with $(0.5-3.0) \mathrm{M}$ hydrochloric, $(2.0-8.0) \mathrm{M}$ nitric, and $(0.1-3.0) \mathrm{M}$ perchloric acids. Hafnium was quantitatively stripped with $(0.5-8.5) \mathrm{M}$ hydrochloric, $(1.0-8.0) \mathrm{M}$ nitric, $(4.0-7.0) \mathrm{M}$ sulfuric and $(1.0-8.0) \mathrm{M}$ perchloric acids. In general, $2 \mathrm{M}$ hydrochloric acid was preferred as the stripping agent, since it facilitated dry ashing before a quantitative analysis.

\section{Separation from binary mixtures}

Zirconium or hafnium was extracted in the presence

Table 3 Effect of the various diluents

\begin{tabular}{lcrrrrr}
\hline \multirow{2}{*}{ Diluent } & \multirow{2}{*}{$\varepsilon$} & \multicolumn{2}{c}{ Zirconium } & & \multicolumn{2}{c}{ Hafnium } \\
\cline { 3 - 4 } \cline { 6 - 7 } & & $\% E$ & \multicolumn{1}{c}{$D$} & & $\% E$ & \multicolumn{1}{c}{$D$} \\
\hline Benzene & 2.28 & 99.0 & 99.0 & & 90.0 & 9.0 \\
Toluene & 2.30 & 99.6 & 249.0 & & 99.6 & 249.0 \\
Xylene & 2.30 & 99.1 & 110.0 & & 99.6 & 249.0 \\
Hexane & 1.89 & 98.0 & 49.0 & & 96.0 & 24.0 \\
Chloroform & 4.80 & 97.0 & 32.0 & & 89.0 & 8.0 \\
CCl $_{4}$ & 2.24 & 60.0 & 1.5 & & 80.0 & 4.0 \\
\hline
\end{tabular}

$\varepsilon$, dielectric constant. of various ions. The tolerance limit was calculated in the usual way. ${ }^{10}$ The alkali and alkaline earths were tolerated in ratios of $1: 125$. The transition elements, however, were tolerated in ratios of $1: 50$; since scandium, molybdenum, uranium, thorium were coextracted, they showed a smaller tolerance limit (Table 5).

Table 5 Effect of diverse ions

\begin{tabular}{|c|c|c|c|}
\hline \multirow{2}{*}{ Element } & \multirow{2}{*}{ Added as } & \multicolumn{2}{|c|}{ Tolerance limit/mg } \\
\hline & & $\mathrm{Zr}$ & Hf \\
\hline $\mathrm{Li}^{+}$ & $\mathrm{LiCl}$ & 5.0 & 5.0 \\
\hline $\mathrm{Na}^{+}$ & $\mathrm{NaCl}$ & 5.0 & 5.0 \\
\hline $\mathbf{K}^{+}$ & $\mathrm{KCl}$ & 5.5 & 5.0 \\
\hline $\mathrm{Be}^{2+}$ & $\mathrm{Be}\left(\mathrm{NO}_{3}\right)_{2} \cdot 3 \mathrm{H}_{2} \mathrm{O}$ & 0.7 & 0.8 \\
\hline $\mathrm{Ca}^{2+}$ & $\mathrm{Ca}\left(\mathrm{NO}_{3}\right)_{2}$ & 5.0 & 5.0 \\
\hline $\mathrm{Mg}^{2+}$ & $\mathrm{MgSO}_{4} \cdot 7 \mathrm{H}_{2} \mathrm{O}$ & 6.0 & 6.0 \\
\hline $\mathrm{Sr}^{2+}$ & $\mathrm{Sr}\left(\mathrm{NO}_{3}\right)_{2} \cdot 2 \mathrm{H}_{2} \mathrm{O}$ & 4.9 & 4.0 \\
\hline $\mathrm{Al}^{3+}$ & $\mathrm{Al}\left(\mathrm{NO}_{3}\right)_{3} \cdot 9 \mathrm{H}_{2} \mathrm{O}$ & 1.0 & 1.0 \\
\hline $\mathrm{Tl}^{3+}$ & $\mathrm{Tl}_{2}\left(\mathrm{SO}_{4}\right)_{3} \cdot 7 \mathrm{H}_{2} \mathrm{O}$ & 2.0 & 2.0 \\
\hline $\mathrm{Zn}^{2+}$ & $\mathrm{ZnSO}_{4} \cdot 7 \mathrm{H}_{2} \mathrm{O}$ & 2.5 & 2.5 \\
\hline $\mathrm{Cd}^{2+}$ & $\mathrm{Cd}\left(\mathrm{NO}_{3}\right)_{2} \cdot 6 \mathrm{H}_{2} \mathrm{O}$ & 2.5 & 2.5 \\
\hline $\mathrm{Cu}^{2+}$ & $\mathrm{CuSO}_{4} \cdot 5 \mathrm{H}_{2} \mathrm{O}$ & 0.9 & 1.0 \\
\hline $\mathrm{Sc}^{3+}$ & $\mathrm{Sc}\left(\mathrm{NO}_{3}\right)_{3}$ & 0.5 & 0.4 \\
\hline $\mathrm{Ce}^{3+}$ & $\mathrm{Ce}\left(\mathrm{NO}_{3}\right)_{3}$ & 1.0 & 1.0 \\
\hline $\mathrm{Y}^{3+}$ & $\mathrm{Y}\left(\mathrm{NO}_{3}\right)_{3}$ & 1.0 & 1.0 \\
\hline $\mathrm{Ti}^{4+}$ & $\mathrm{Ti}\left(\mathrm{SO}_{4}\right)_{2}$ & 0.25 & 0.4 \\
\hline $\mathrm{V}^{5+}$ & $\mathrm{NH}_{4} \mathrm{VO}_{3}$ & 0.3 & 0.4 \\
\hline $\mathrm{Co}^{2+}$ & $\mathrm{CoCl}_{2} \cdot 6 \mathrm{H}_{2} \mathrm{O}$ & 0.8 & 0.9 \\
\hline $\mathrm{Cr}^{3+}$ & $\mathrm{Cr}\left(\mathrm{NO}_{3}\right)_{3}$ & 0.9 & 0.8 \\
\hline $\mathrm{Cr}^{6+}$ & $\mathrm{K}_{2} \mathrm{Cr}_{2} \mathrm{O}_{7}$ & 0.8 & 1.2 \\
\hline $\mathrm{Mo}^{6+}$ & $\left(\mathrm{NH}_{4}\right)_{6} \mathrm{Mo}_{7} \mathrm{O}_{24} \cdot 4 \mathrm{H}_{2} \mathrm{O}$ & 0.5 & 0.5 \\
\hline $\mathrm{UO}^{2+}$ & $\mathrm{UO}_{2}\left(\mathrm{NO}_{3}\right)_{2} \cdot 6 \mathrm{H}_{2} \mathrm{O}$ & 0.3 & 0.2 \\
\hline $\mathrm{Th}^{4+}$ & $\mathrm{Th}\left(\mathrm{NO}_{3}\right)_{4}$ & 0.4 & 0.5 \\
\hline $\mathrm{Hf}^{4+}$ & $\mathrm{Hf}\left(\mathrm{NO}_{3}\right)_{4}$ & 0.4 & - \\
\hline $\mathrm{Zr}^{4+}$ & $\mathrm{Zr}\left(\mathrm{NO}_{3}\right)_{4}$ & - & 0.4 \\
\hline $\mathrm{Cl}^{-}$ & $\mathrm{NaCl}$ & 1.0 & 1.0 \\
\hline $\mathrm{SO}_{4}{ }^{2-}$ & $\mathrm{Na}_{2} \mathrm{SO}_{4}$ & 1.0 & 1.0 \\
\hline $\mathrm{NO}_{3}^{-}$ & $\mathrm{NH}_{4} \mathrm{NO}_{3}$ & 3.0 & 3.0 \\
\hline $\mathrm{SeO}_{3}^{2-}$ & $\mathrm{Na}_{2} \mathrm{SeO}_{3}$ & 0.8 & 0.8 \\
\hline $\mathrm{TeO}_{3}{ }^{2-}$ & $\mathrm{Na}_{2} \mathrm{TeO}_{3}$ & 0.8 & 0.8 \\
\hline $\mathrm{Cit}^{3-}$ & citric acid & 1.0 & 1.5 \\
\hline $\operatorname{Tart}^{3-}$ & tartaric acid & 2.0 & 2.0 \\
\hline $\mathrm{Mal}^{2-}$ & malonic acid & 2.0 & 2.0 \\
\hline
\end{tabular}

Table 4 Effect of the stripping agent concentration on the \%recovery

\begin{tabular}{|c|c|c|c|c|c|c|c|c|}
\hline \multirow{2}{*}{ Conc./M } & \multicolumn{2}{|c|}{$\mathrm{HCl}$} & \multicolumn{2}{|c|}{$\mathrm{HNO}_{3}$} & \multicolumn{2}{|c|}{$\mathrm{H}_{2} \mathrm{SO}_{4}$} & \multicolumn{2}{|c|}{$\mathrm{HClO}_{4}$} \\
\hline & $\mathrm{Zr}$ & Hf & $\mathrm{Zr}$ & Hf & $\mathrm{Zr}$ & Hf & $\mathrm{Zr}$ & Hf \\
\hline 0.1 & 90.0 & - & 89.0 & 90.0 & - & - & 98.0 & 96.0 \\
\hline 0.5 & 99.0 & 99.0 & 90.0 & 98.0 & 0 & 0 & 99.6 & 98.0 \\
\hline 1.0 & 99.6 & 99.6 & 98.4 & 99.0 & 45.0 & 40.0 & 99.6 & 99.0 \\
\hline 2.0 & 99.6 & 99.6 & 99.6 & 99.6 & 63.0 & 95.0 & 99.6 & 99.4 \\
\hline 4.0 & 89.6 & 99.6 & 99.6 & 99.6 & 45.0 & 99.6 & 98.0 & 99.5 \\
\hline 6.0 & 52.0 & 99.6 & 99.6 & 99.6 & 35.0 & 99.6 & 90.0 & 99.6 \\
\hline 8.0 & 10.0 & 99.6 & 99.6 & 98.0 & 35.0 & 98.0 & 80.0 & 99.0 \\
\hline 9.5 & 0.0 & 99.0 & - & - & - & - & - & - \\
\hline
\end{tabular}


Table 6 Separation from multicomponent mixtures

\begin{tabular}{|c|c|c|c|c|c|c|c|}
\hline & Metal & Taken/ $\mu \mathrm{g}$ & Found $/ \mu \mathrm{g}$ & Stripping agent/M & $\%$ Recovery & Chromogenic ligand & $\lambda_{\max } / \mathrm{nm}^{12}$ \\
\hline \multirow[t]{4}{*}{1} & Ti(IV) & 100 & 96.0 & $0.1 \mathrm{H}_{2} \mathrm{SO}_{4} 3 \% \mathrm{H}_{2} \mathrm{O}_{2}$ & 96.0 & Tiron & 380 \\
\hline & Th & 50 & 48.6 & $0.5 \mathrm{H}_{2} \mathrm{SO}_{4}$ & 95.8 & Arsenazo III & 650 \\
\hline & Hf & 40 & 39.1 & $9 \mathrm{HCl}$ & 99.1 & Xylenol Orange & 540 \\
\hline & $\mathrm{Zr}$ & 20 & 19.8 & $2 \mathrm{HCl}$ & 99.0 & Arsenazo III & 650 \\
\hline \multirow[t]{3}{*}{2} & $\mathrm{Zr}$ & 40 & 39.6 & $6 \mathrm{HCl}$ & 99.6 & Arsenazo III & 650 \\
\hline & In & 80 & 79.4 & $1 \mathrm{HNO}_{3}$ & 99.6 & PAN & 540 \\
\hline & $\mathrm{Al}$ & 150 & 149.0 & - & 99.0 & Alizarin Red S & 520 \\
\hline \multirow[t]{3}{*}{3} & $\mathrm{Hf}$ & 100 & 99.6 & $6 \mathrm{HCl}$ & 99.6 & Xylenol Orange & 540 \\
\hline & Mo(VI) & 100 & 99.1 & $1 \mathrm{HNO}_{3}$ & 99.1 & Tiron & 380 \\
\hline & $\mathrm{Cr}(\mathrm{III})$ & 200 & 199 & - & 98.0 & sym-diphenyl carbazide & 570 \\
\hline \multirow[t]{3}{*}{4} & Sc & 80 & 79.4 & $0.5 \mathrm{H}_{2} \mathrm{SO}_{4}$ & 99.6 & Arsenazo III & 670 \\
\hline & $\mathrm{Z}_{\mathbf{r}}$ & 40 & 39.6 & $2 \mathrm{HCl}$ & 99.6 & Arsenazo III & 650 \\
\hline & $\mathrm{Y}$ & 100 & 99.0 & - & 99.6 & Arsenazo III & 660 \\
\hline \multirow[t]{3}{*}{5} & Hf & 40 & 39.6 & $8 \mathrm{HNO}_{3}$ & 99.6 & Xylenol Orange & 540 \\
\hline & $\mathrm{U}(\mathrm{VI})$ & 100 & 99.6 & $0.5 \mathrm{NaOH}$ & 99.6 & Arsenazo III & 665 \\
\hline & $\mathrm{Fe}(\mathrm{III})$ & 250 & 248 & - & 99.2 & 1,10-phenanthroline & 510 \\
\hline \multirow[t]{4}{*}{6} & $\mathrm{Zr}$ & 40 & 39.6 & $8 \mathrm{HNO}_{3}$ & 99.6 & Arsenazo III & 650 \\
\hline & Th & 80 & 79 & $5 \mathrm{HCl}$ & 99.0 & Arsenazo III & 650 \\
\hline & $\mathrm{U}(\mathrm{VI})$ & 100 & 99.0 & $0.5 \mathrm{NaOH}$ & 99.0 & Arsenazo III & 665 \\
\hline & $\mathrm{Al}$ & 150 & 149.0 & - & 99.0 & Alizarin Red S & 510 \\
\hline \multirow[t]{4}{*}{7} & $\operatorname{Ti}(\mathrm{IV})$ & 100 & 96.4 & $0.5 \mathrm{H}_{2} \mathrm{SO}_{4}$ & 99.6 & Tiron & 380 \\
\hline & $\mathrm{Hf}$ & 40 & 39.4 & $9 \mathrm{HCl}$ & 98.6 & Xylenol Orange & 540 \\
\hline & $\mathrm{Zr}$ & 20 & 19.2 & $2 \mathrm{HCl}$ & 98.4 & Arsenazo III & 650 \\
\hline & $\mathrm{Fe}(\mathrm{III})$ & 250 & 249 & - & 99.6 & 1,10-phenanthroline & 510 \\
\hline
\end{tabular}

\section{Separation of zirconium and hafnium}

Although zirconium and hafnium were coextracted, there was a significant difference in their stripping behaviors. Thus, when hafnium was stripped with $9 \mathrm{M}$ hydrochloric acid, zirconium was not stripped at all. It was stripped later with $2 \mathrm{M}$ hydrochloric acid. For such a separation, the separation factor was quite large. At $2 \mathrm{M}$ hydrochloric acid, the distribution ratio for both metals was 999; at $9 \mathrm{M}$ hydrochloric acid the distribution ratio of zirconium was 0 , while that of hafnium was 999 . Consequently, the separation factor $(\alpha)$ was $\infty$ facilitating their separation (Fig. 3).

Similarly, the sequential separation of titanium, zirconium, hafnium, and thorium was accomplished after coextraction by stripping titanium with $0.1 \mathrm{M}$ sulfuric acid containing $10 \%(\mathrm{v} / \mathrm{v})$ of hydrogen peroxide, then thorium with $0.5 \mathrm{M}$ sulfuric acid, hafnium with $9 \mathrm{M}$ hydrochloric acid, and finally zirconium with $2 \mathrm{M}$ hydrochloric acid (Table 6).

\section{Separation from multicomponent mixtures}

A mixture of zirconium, indium and aluminum was separated by stripping zirconium with $6 \mathrm{M}$ hydrochloric acid, and indium with $1 \mathrm{M}$ nitric acid when aluminum was not extracted. The mixture of hafnium, molybdenum and chromium(III) was separated by stripping hafnium with $6 \mathrm{M}$ hydrochloric acid, and molybdenum with $1 \mathrm{M}$ nitric acid when chromium was not extracted. Scandium, zirconium and yttrium were

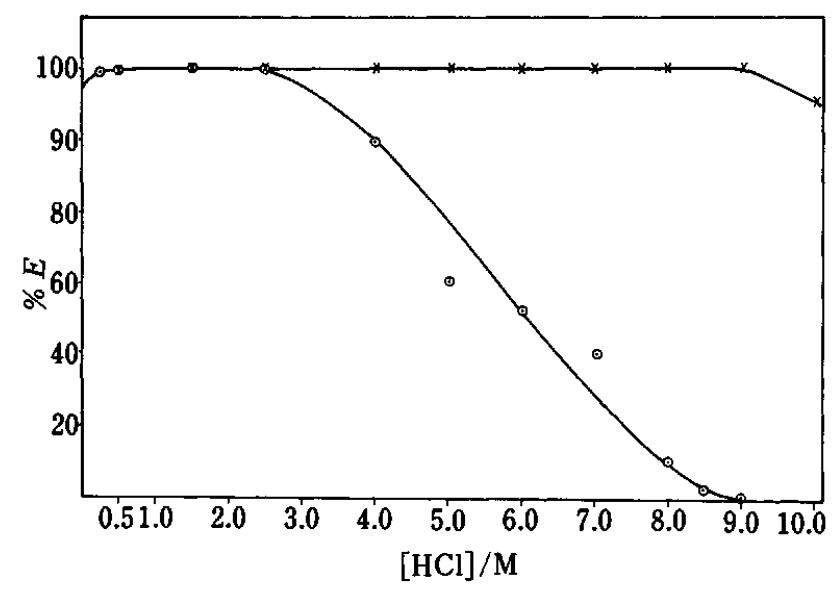

Fig. $3 \%$ Recovery of zirconium and hafnium at various concentrations of hydrochloric acid. $\times$, hafnium; $\odot$, zirconium.

separated by stripping scandium with $0.5 \mathrm{M}$ sulfuric acid, and zirconium with $2 \mathrm{M}$ hydrochloric acid when yttrium was not extracted. The separation of hafnium, uranium (VI) and iron(III) was effected by stripping hafnium with $8 \mathrm{M}$ nitric acid, and uranium with $0.5 \mathrm{M}$ sodium hydroxide, when iron was not extracted.

The quaternary mixture of zirconium, thorium, uranium and aluminum was separated by stripping zirconium with $8 \mathrm{M}$ nitric acid, thorium with $5 \mathrm{M}$ hydro- 
chloric and, finally, uranium with $0.5 \mathrm{M}$ sodium hydroxide, when aluminum was not extracted. A mixture of titanium, zirconium, hafnium, and iron(III) was separated by stripping titanium with $0.5 \mathrm{M}$ sulfuric acid, hafnium with $9 \mathrm{M}$ hydrochloric acid, and zirconium with $2 \mathrm{M}$ hydrochloric acid when iron was not extracted (Table 6).

The total time required for the separation and determination was just $30 \mathrm{~min}$. The significant features concerning this method are the sequential separation of titanium, zirconium, thorium and hafnium. Separation from chromium, vanadium, uranium and molybdenum is important since they are associated in minerals. This method is simple, rapid, selective and reproducible. The relative standard deviation is $\pm 1.2 \%$.

The authors are thankful to the Council of Scientific and Industrial Research for sponsoring this project and awarding a Senior Research Fellowship to one of them (MAK).

\section{References}

1. J. Hure, M. Rastoix, R. Saint-James, Anal. Chim. Acta, 25, 1 and 118 (1961)

2. W. Fischer, B. Deierling, H. Meitsch, G. Otto, H. P.
Pohlmann and K. Reinhardt, Angew. Chem., Int. Ed. Engl., 5, 15 (1966).

3. J. Liu and A. Chow, Talanta, 34, 331 (1987).

4. A. K. De, S. M. Khopkar and R. A. Chalmers, "Solvent Extraction of Metals", Van Nostrand Reinhold, London, 1970.

5. E. Cerrai and C. Testa, Energia Nuclie, 6, 707 and 768 (1959); Chem. Abstr., 54, 10713h.

6. I. S. El-Yamani, M. Y. Farah and F. A. Abd El-Aleim, Talanta, 25, 534 (1978).

7. R. R. Rao and S. M. Khopkar, Indian J. Chem., 23A, 504 (1984).

8. C. P. Vibhute and S. M. Khopkar, Anal. Chim. Acta, 193, 387 (1987).

9. N. M. Sundaramurthi and V. M. Shinde, Analyst [London], 114, 201 (1989).

10. M. A. Karve and S. M. Khopkar, Anal. Sci., 8, 77 (1992).

11. A. K. Mukherji, "Analytical Chemistry of Zirconium and Hafnium", pp. 51, 23, 75, 76, Pergamon Press, Oxford, 1970.

12. F. D. Snell, "Photometric and Fluorimetric Methods of Analysis", Part I and II, Wiley Interscience, New York, 1978.

(Received July 15, 1991)

(Accepted December 28, 1991) 